\title{
A Sectoral Hierarchical Clustering of SMEs in Turkey with Respect to General Support Programs
}

\author{
Ali Kemal Çelik ${ }^{1}$, Emrah Talaş ${ }^{2} \&$ Ahmet İlker Akbaba ${ }^{1}$ \\ ${ }^{1}$ Department of Business Administration, Atatürk University, Erzurum, Turkey \\ ${ }^{2}$ Department of Econometrics, Atatürk University, Erzurum, Turkey \\ Correspondence: Ali Kemal Çelik, Faculty of Economics and Administrative Sciences, Department of Business \\ Administration, Atatürk University, Erzurum 25240, Turkey. Tel: 90-442-231-2099. E-mail: \\ akemal.celik@atauni.edu.tr
}

Received: July 5, 2013 Accepted: August 28, 2013 Online Published: October 11, 2013

doi:10.5539/res.v5n5p84 URL: http://dx.doi.org/10.5539/res.v5n5p84

This paper is a revised and extended version of the conference paper presented at "9th Annual International Conference on Small and Medium Sized Enterprises: Management-Marketing-Economic Aspects", on 30-31 July \& 1-2 August 2012, Athens, Greece.

\begin{abstract}
Small and Medium-Sized Enterprises (SMEs) have to be supported by appropriate programs, especially in developing countries. The Small and Medium Enterprises Development Organization (KOSGEB) is the institution in Turkey that assists SMEs to survive in domestic and global markets by providing support programs. Effective and efficient management of support programs by considering sectoral requirements is thus crucial for the success and survival of SMEs. This study uses hierarchical clustering analysis to investigate the success of the general support programs which KOSGEB provides in order to assist SMEs in Turkey on the purpose of playing more prominent roles in domestic and global markets. Hierarchical clustering analysis is a multivariate technique for building a binary tree of data and visualizing it in summary form. Using hierarchical clustering analysis, the general support programs for SMEs in Turkey were summarized with respect to sector for the years 2010 and 2011. Ward's hierarchical analysis was used to analyze the data. The results of the corresponding analyses suggest that the sectors of SMEs in Turkey generally agglomerate in two or three clusters for both years. KOSGEB might consider the similarities of these sectors to draw greater advantage from its general support programs and look for new opportunities to encourage these sectors by providing these programs more efficiently.
\end{abstract}

Keywords: SME, support programs, hierarchical clustering analysis, KOSGEB, Turkey

\section{Introduction}

Small- and Medium-Sized Enterprises (SMEs) today make a crucial contribution to all economies worldwide. Developing countries are particularly cognizant of the importance of SMEs, since SMEs account for nearly all of the enterprise sectors in such countries, and provide more than two-thirds of total employment. However, the success and survival of SMEs is no simple matter. The challenges faced by SMEs must be taken into consideration by all levels of government, and they should be provided with suitable financial support and strategic guidance, especially in their early stages of operation. Such tasks are generally arranged by governments, which commonly delegate their authority to smaller professional organizations or institutions. In Turkey, the Small and Medium Enterprises Organization (KOSGEB) is the authorized institution for supporting Turkish SMEs by integrating them into competitive markets, and increasing their efficiency and competitive potential both domestically and globally. General support programs are the most utilized functions provided by KOSGEB, and sectoral investigation of these programs in terms of SMEs can be instructive for achieving goals both of SMEs and KOSGEB itself.

The degree of concentration of SMEs in the same sector is not currently considered to be optimal in Turkey, and as the competitive advantage of SMEs has become important for the global economy, clustering policies now have a role in most Turkish development plans. Numerous institutions and strategic alliances have in fact been 
set up by governments in order to achieve global and local economic success. As the popularity of clustering has increased, researchers have turned their attention to this phenomenon. Hierarchical clustering analysis, one of the most frequently used methods in the social science literature, here proves to be very helpful. This is a multivariate statistical technique for building a binary tree and visualizing it in summary form. Such analysis is useful for agglomerating groups or cases into sub-clusters when the natural number of clusters is unpredictable. These sub-clusters are determined with respect to similarity and dissimilarity measures. Forming new clusters by their similarities and monitoring the dissimilarities of groups or cases is a means to illustrate the existing position with respect to the research area.

In this study, a sectoral hierarchical clustering analysis of SMEs in Turkey was performed with respect to KOSGEB's general support programs for the years 2010 and 2011. This analysis could be a guide for both SMEs, and KOSGEB itself, as they seek to become competitive in the domestic and global markets.

\section{The Concepts of Clusters and Clustering}

According to Michael E. Porter, clusters are geographic concentrations of interconnected companies and institutions in a particular field, and they encompass an array of linked industries and other entities important to competition (Porter, 1990). Clusters are a part of a broader conceptual framework for understanding the drivers of regional and national competitiveness. This framework, grounded in the analysis provided in Porter's The Competitive Advantage of Nations (1990), provides a connection between firm-level behavior and economic policy at the micro as well as the macroeconomic level (Ketels \& Sölvell, 2006). Starting a cluster involves, first, building the economical fundamentals for an industry or technology, and, second, finding the spark of entrepreneurship required to get it going. Both of these are supported by a number of common elements in the regions that we examined (Bresnahan et al., 2005). The manner in which clusters emerge and evolve is important for public policy and corporate strategy. Understanding how and why clusters emerge and develop provides insights into agglomeration phenomena, innovation capacity, and location advantages, and may influence local government investment (Ferreira et al., 2006).

The concept of clusters focuses on the profits that firms accrue due to their connections with or proximity to other firms. These profits result from cooperation, market relations, spillovers, and in some cases the fact that more start-ups occur. Most of these processes, especially spillovers and increased frequency of start-ups, are much more effective locally; thus, clusters are often a local phenomenon (Brenner, 2005). The boundaries of a cluster do not generally conform to ordinary administrative borders such as municipalities, counties, or even countries. Clusters are dynamic, with boundaries in constant change as new companies and new linkages appear and others disappear (Nordin, 2003). The geographic scope of clusters can vary from a single city, state, or region, to a network of companies across state or even country borders. There are various forms that may help to optimize competitive advantage in the development of clusters (Braun et al., 2005). Although each existing local industrial cluster has its own specific history, clustering is a phenomenon that is common for many industries and for different periods in the history of industrialized production. The contours of local industrial clusters are based on the mechanisms that cause their emergence and persistence (Brenner, 2005).

Clustering policy generally concentrates on SMEs. One or more big companies generally dominate a cluster; however, even where a big company has a directive role, SMEs are essential structural elements of the cluster itself (White Paper, 2008). Studies conducted across the world suggest that there are three basic elements of successful clusters: (a) the presence of an internationally active firm which dominates the market and generates technology, (b) suppliers and complementary businesses (mostly SMEs), (c) specialists based upon innovative and dynamic information (Kaplan, 2009). In many developing countries, SME agglomerations are a widespread phenomenon. On the outskirts of many cities, significant numbers of micro- and small-scale enterprises often operate close to one another and produce similar goods. While clusters of this kind are common in metalworking, woodworking, and textiles, few of them have the virtues of successful cluster models (UNIDO programme, 2001).

\section{Clustering Attempts in Turkey and in the World}

Clustering surveys in Turkey began in 1999 with the Competitive Advantage of Turkey (CAT) platform, which was formed through the contributions of the Middle East Strategy Center (under the guidance of Michael Porter) and the Turkish private sector. The platform was later transformed into the National Competitiveness Research Institute, and thereby institutionalized. After the stage of early analysis and exploratory studies, the clustering and development of clusters was accelerated with the aid of an assignment of funds from the European Union.

The "Development of National Clustering Policy" project, one of the most notable projects concerned with clustering in Turkey, started in March 2007 with financial support from the European Commission. The project 
aimed to position Turkey - uniquely in the world - as a country which possesses a national clustering policy. In this context it developed national administrative and constitutional capacity, and the results of the project were considered to comprise a national strategy. Within the scope of the project, an inter-institutional working group, consisting of sixteen stakeholders from governmental, scientific, and academic institutions, was constituted for cooperation (White Book, 2008). The corporate structures associated with clustering in Turkey before this project was initiated are summarized in Table 1.

Ongoing work on clustering and networking in Turkey is being carried out under the SME Coordination and Clustering Project, which began in February 2011 and continues until August 2013. The project is being carried out and financed as a collaboration between of the Ministry of Economy, the Ministry of Science, Industry, and Technology, ECORYS Turkey, and the European Union. The aim of the project is to guide target sectors via interventions from clustering information centers constituted in five provinces (Çorum, Gaziantep, Kahramanmaraş, Samsun, \& Trabzon). Within the framework of these clustering centers, SMEs in Turkey are intended to achieve the following:

- acquiring technical information required for exportation

- $\quad$ easier monitoring of global markets

- technical support regarding differentiation of product and service, innovation, and development of staff

- providing for information sharing by creating collaboration opportunities with domestic and international companies

- entering international markets and providing opportunities for monitoring technology to maintain export volume. (SME Networking Project, 2011)

Table 1. Corporate structures in turkey related to clustering

\begin{tabular}{|c|c|c|}
\hline $\begin{array}{l}\text { Corporate } \\
\text { Structure }\end{array}$ & Conceptual Definition & $\begin{array}{l}\text { Related Corporate Structures } \\
\text { in context of Turkey }\end{array}$ \\
\hline $\begin{array}{l}\text { Regional } \\
\text { Agglomeration }\end{array}$ & $\begin{array}{l}\text { Agglomeration for related firms of the same } \\
\text { sector in the same region geographically without } \\
\text { neccessity }\end{array}$ & $\begin{array}{ll}\text { - } & \text { Organized Industry Regions } \\
& \text { (OIR) } \\
\text { - } & \text { Specialized OIR Free Trade } \\
& \text { Regions } \\
\text { - } & \text { Technoparks } \\
\text { - } & \text { Adiyaman Ready-Made } \\
& \text { Clothing Clustering Project } \\
\text { - Şanliurfa Ready-Made }\end{array}$ \\
\hline Clustering Projects & $\begin{array}{l}\text { Clustering and relatedness of actors in a particular } \\
\text { profession, region complementarily }\end{array}$ & $\begin{array}{l}\text { Clustering Project } \\
\text { - Fashion and Textile } \\
\text { Clustering Project } \\
\text { - Bartın Shipping Industry } \\
\text { Clustering Project } \\
\text { - Çukurova Clustering } \\
\text { Project, regional innovation } \\
\text { system } \\
\text { - Textile Industry in Istanbul, } \\
\text { Bursa and Denizli }\end{array}$ \\
\hline $\begin{array}{l}\text { Industrial and } \\
\text { Tertiary Networks }\end{array}$ & $\begin{array}{l}\text { Assembling of actors in a particular industry area } \\
\text { as "information or production networks" without } \\
\text { geographical similarity or complementarity }\end{array}$ & $\begin{array}{l}\text { - Furniture Industry in } \\
\text { - Ankara, Kayseri and Bursa } \\
\text { Automotive Industry in } \\
\text { Bursa and Kocaeli } \\
\text { - } \quad \text { Wine production in } \\
\text { Nevşehir and Tekirdağ } \\
\text { - Service Sector Networks } \\
\text { (medical, tourism and } \\
\text { logistic) }\end{array}$ \\
\hline
\end{tabular}

Source: White Book Development of a Clustering Policy for Turkey (2008). 
Alongside this work, there are many references in the macroeconomic documents provided by the Turkish government concerning supporting clustering and clustering projects. For instance, under the topic "Increasing Competitive Power" of the ninth Development Planning, promulgated by Prime Ministry State Planning Organization, we can find statements such as "Physical infrastructure requirements of businesses will be satisfied and their networking and clustering attempts will be supported," and similar statements about clustering under the topic "Establishing Regional Development" (Kaplan, 2009). These strongly demonstrate governmental support for clustering policies.

Many studies in the literature have emphasized the vital role of the SME clusters with respect to empirical evidence. For instance, Altenburg and Meyer-Stamer (1999) proposed to differentiate and promote between three types of clusters in Latin America. According to the authors, survival clusters of micro-and small-scale enterprises owe their existence more to unfavorable macroeconomic conditions and less to entrepreneurial competence and dynamism. The main challenge in the clusters for more advanced and differentiated mass producers is to create an environment that supports learning, innovation, and constant upgrading. Finally, clusters of transnational corporations often are showcases of best-practice manufacturing. Visser (1999) investigated the strength and type of clustering advantages, contrasting the performance of small clustered firms with several control groups of dispersed producers in Peru based on the empirical evidence. He suggested that clustering advantages were significant for the smallest firms and cost reductions and information spillovers were the dominant type of these advantages. Wever and Stam (1999) examined the relevance of high technology clusters in the specific Dutch context. They exhibited most high technology SMEs had nationwide linkages. Berry et. al. (2001) discussed the SMEs in Indonesia before and during the crisis and the prevalance of SME clusters suggested that they benefit for SME development. Keeble and Nachum (2002) analyzed the results of a detailed survey of 300 small and medium-sized management and engineering consultancies in London and southern England and particular attention was paid to the notion of clustering, the role of demand-side influences, localized collective learning processes, and increasing globalization in clustering. Rocha and Sternberg (2005) investigated the impact of clusters on entrepreneurship using 97 German planning regions as units of analysis and they suggested that clusters had an impact on entrepreneurship even though industrial agglomerations had no impact. Chen and Cao (2006) described the character of Chinese SME clusters, which were highly concentrated both geographically and by industrial sector and they suggested that product structure, a favourable innovation environment, learning capability, and enthusiasm for innovation were crucial for innovation capabilities in the corresponding clusters. Shapira (2008) examined the evolving situation of mature manufacturing SMEs in Japan and the United States and considered some of the key challenges they face in an era of globalization and rapid technological change. Consequently, a review was offered of illustrative policy initiatives in three areas including framework policies, industrial services, and regional industrial cluster policies.

\section{Cluster Analysis}

Cluster analysis is the generic name for a wide variety of procedures that can be used to create a classification scheme. These are empirical procedures for forming "clusters" or groups of highly similar entities. More specifically, a clustering method is a multivariate statistical procedure that starts with a data set containing information about a sample of entities and attempts to reorganize these entities into relatively homogeneous groups (Aldenderfer \& Blashfield, 1984). The general purpose of clustering analysis is to classify ungrouped data with regards to their similarities (Tatlidil, 2002). The groups or clusters should be as homogeneous as possible and the differences among the various groups as large as possible (Härdle \& Simar, 2007). In addition, clustering analysis is used for purposes such as the determination of actual types, forecasting of groups, hypothesis testing, assessment of clusters, and establishing outliers (Kalayc1, 2006).

Aldenderfer and Blashfield (1984) summarize clustering analysis methods as having five basic steps:

(1) selection of a sample to be clustered

(2) definition of a set of variables on which to measure the entities in the sample

(3) computation of the similarities among the entities

(4) use of a cluster analysis method to create groups of similar entities

(5) validation of the resulting cluster solution.

Clustering algorithms partition data objects (patterns, entities, instances, observances, units) into a certain number of clusters (groups, subsets, or categories) (Xu \& Wunsch II, 2009). There are essentially two types of clustering methods: hierarchical algorithms and partitioning algorithms. The hierarchical algorithms can be divided into agglomerative and splitting procedures. Agglomerative hierarchical clustering starts from the finest 
partition possible (each observation forms a cluster) and groups them (Härdle \& Simar, 2003). These are a class of clustering techniques that proceed by a series of steps in which progressively larger groups are formed by joining together groups formed earlier in the process. The initial step involves combining the two individuals which are closest (according to whatever distance measure is being used). The process goes from individuals to a final stage in which all individuals are combined, with the closest two groups being combined at each stage. The series of steps in this type of clustering can be conveniently summarized in a tree-like diagram known as a dendogram (Landau \& Everitt, 2004). The observations are listed on the horizontal axis and the vertical axis represents the Euclidean distance between clusters. In order to determine the cluster composition for a given number of clusters the dendogram can be cut at the appropriate place. Different criteria can be used for determining the best number of clusters (Sharma, 1996).

\section{Hierarchical Clustering Methods}

Some of the popular hierarchical clustering methods are: nearest neighbor or single-linkage method, farthest-neighbor or complete linkage method, centroid method, average-linkage method, and Ward's method. The first step is the same for all the methods, but after the first step the methods differ with respect to the procedure used to compute the distances between clusters (Sharma, 1996).

\subsection{Single Linkage Method}

The single linkage method, described by Sneath (1957), forms clusters by the following rule: Cases will be joined to existing clusters if at least one of the members of the existing cluster is of the same level of similarity as the case under consideration for inclusion. The distance between two clusters is represented by the minimum of the distance between all possible pairs of subjects in the two clusters (Sharma, 1996). Single linkage begins the clustering process by searching for the two most similar entities in the matrix. The major advantage of this method is that it is invariant to monotonic transformations of the similarity matrix, and is unaffected by ties in the data. This means that single linkage is one of the few methods that will not affected by any data transformation that retains the same relative ordering of values in the similarity matrix (Aldenderfer \& Blashfield, 1984). If the clusters are separated far from each other, the single linkage method works well (Xu \& Wunsch II, 2009).

\subsection{Complete Linkage Method}

The complete linkage method was put forward by several researchers working independently and studying in different time periods. Horn (1943) used this method to cluster test variables; Sorensen (1948) developed the method for ecological studies; and McQuitty (1961) recommended that species analysis be undertaken by this method (Yaylalı et al., 2006). This method is the logical opposite of single linkage clustering in that the linkage rule states that any candidate for inclusion into an existing cluster must fall within a certain level of similarity to all members of that cluster (Aldenderfer \& Blashfield, 1984). The distance between two clusters is defined as the maximum of the distances between all possible pairs of observations in the two clusters (Sharma, 1996). The complete linkage method has a more rigorous rule than single linkage, and, therefore, complete linkage has a tendency to find relatively compact hyperspherical clusters composed of highly similar cases (Aldenderfer \& Blashfield, 1984).

\subsection{Average Linkage Method}

The average linkage method was proposed by Sokal and Michener (1958), and average linkage clustering was developed as an antidote to the extremes of both single and complete linkage. Although there are a number of variants of the method, essentially each computes an average of the similarity of a case under consideration with all cases in the existing cluster (Aldenderfer \& Blashfield, 1984). In the average linkage method the distance between two clusters is obtained by taking the average distance between all pairs of subjects in the two clusters (Sharma, 1996). For average linkage, the distance between two clusters is found by computing the average dissimilarity of each item in the first cluster to each item in the second cluster (Izenman, 2008).

\subsection{Centroid Method}

In the centroid method each group is replaced by an average subject which is the centroid of that group. In other words, centroids are changed when a new candidate is involved in a cluster. This method is less affected by outliers as compared to other hierarchical clustering methods (Çokluk et al., 2010). The distance between two clusters $A$ and $B$ is defined as the Euclidean distance between the mean vectors (often called centroids) of the two clusters

$$
D(A, B)=d\left(\bar{y}_{A}, \bar{y}_{B}\right)
$$


where $\bar{y}_{A}$ and $\bar{y}_{B}$ are the mean vectors for the observation vectors in $A$ and the observation vectors in $B$, respectively (Rencher, 2002).

\subsection{Ward's Method}

Ward's method was proposed to optimize the minimum variance in a cluster (Ward, 1963). This method is also known as the sum of in-group squares or sum of squared errors. Wishart (1969) has indicated how Ward's method can be applied by using Euclidean matrices in between centroid clusters (Yaylali, 2006). The object of Ward's method is to minimize the increase of the within-class sum of the squared errors,

$$
E=\sum_{k=1}^{K} \sum_{x_{j}} \epsilon c_{k}\left\|x_{t}-m_{k}\right\|^{2}
$$

where $K$ is the number of clusters and $m_{k}$ is the centroid cluster $C_{k}$, caused by the merger of two clusters. This change is only computed on the formed cluster and the two clusters to be merged, and can be represented as

$$
\Delta E_{i j}=\frac{n_{i} n_{j}}{n_{i}+n_{j}}\left\|m_{i}-m_{j}\right\|^{2}
$$

where $n_{i}$ is the number of data points belonging to the cluster (Xu \& Wunsch II, 2009). As the formula shows, the minimum increase of the sum of squared errors is directly proportional to the Euclidean distance in between the centers of merged clusters (Yaylali et al., 2006).

The splitting procedure starts with the coarsest partition possible: one cluster contains all of the observations. It proceeds by splitting the single cluster up into smaller-sized clusters. The partitioning algorithms start from a given group definition and proceeds by exchanging elements between groups until a certain score is optimized. The main difference between the two clustering techniques is that in hierarchical clustering, once groups are found and elements are assigned to the groups, this assignment cannot be changed. In partitioning techniques, on the other hand, the assignment of objects into groups may change during the application of the algorithm (Härdle \& Simar, 2003).

Hierarchical agglomerative methods have been dominant among the families of methods in terms of frequency of their applied use (Aldenderfer \& Blashfield, 1984). In contrast to other types of cluster analysis in which a single set of mutually exclusive and exhaustive clusters is formed, hierarchical clustering analysis technique proceeds sequentially from tighter, less inclusive clusters through larger more inclusive clusters and is continued until all variables are clustered in a single group (Bridges, 1966).

\section{Methodology and Data Set}

This study performs a sectoral hierarchical clustering of SMEs in Turkey with respect to the general support programs provided by KOSGEB in 2010 and 2011. The data set was obtained from the database of the KOSGEB headquarters in Ankara and the amount of general support programs in Turkish liras were analyzed based on particular supported sectors. Although all of the hierarchical methods in the literature have pros and cons, this study selected Ward's hierarchical clustering method in order to attain a picture of the sectors with respect to the general programs. Since hierarchical clustering analysis is more appropriate when the sample size is typically smaller than 250 (Çokluk et al., 2010) and the number of clusters is unpredictable - as is the data with which we are concerned in this study - it was considered that this clustering method would have the optimal results with respect to the our intentions. KOSGEB declares its vision as "to be the institution that provides SMEs to have the right to comment in the global market, effective in determining SME and entrepreneurship policies, and taken as a model in all over the model" (Republic of Turkey Small and Medium Enterprises Development Organization, 2013). In that visional context, the importance of SME clustering policies revisits and the motivation of this study is to provide empirical evidence of general support programs for Turkish SMEs in a hierarchical clustering approach. The general support programs were preliminarily performed by KOSGEB in the corresponding years, thus the study also employs to exhibit the very first application of these programs, and thereby highlights various decision makers including the government, KOSGEB and Turkish SMEs.

The agglomerative hierarchical algorithm and the squared Euclidean distance measure were used for Ward's hierarchical method in this study because of their efficiency, as well as their familiarity from the social sciences. When the differences among means and variances of variables on data matrices are erratic, overvalued variables have a dramatic effect on the roles of other variables. In addition, extreme values of variables have a negative effect on clusters. In such circumstances, the data should be standardized or transformed (Özdamar, 1999). In this study, the data were standardized by via Z-score transformation to eliminate the problematic negative effects 
of extreme values (outliers). A normally distributed experimental result $\mathbf{X}$ is standardized by using the following formula:

$$
Z=\frac{X-\bar{X}}{S}
$$

where $\bar{X}$ denotes the mean and $S$ denotes the standard deviation. The normal distribution assumption was tested for the data by using the one-sample Kolmogorov-Smirnov test and it was determined that the data satisfied the normal distribution requirement at the 0.05 significance level (p-values were greater than 0.05). Various distance measures can be used to form hierarchical clusters, such as Euclidean distances, squared Euclidean distances, Manhattan distance (city-block metric), and the Chebychev distance measure (Yaylali et al., 2006). In this study, squared Euclidean distances were used. The most common distance measure method is the Euclidean distance given by

$$
d_{i j}=\left[\sum_{l=1}^{q}\left(x_{i l}-x_{j l}\right)^{2}\right]^{\frac{1}{2}}
$$

where $d_{i j}$ is the Euclidean distance for two individuals $i$ and $j$, each measured on $q$ variables, $x_{i l}, x_{j l}, l=1, \ldots$, $q$ (Landau \& Everitt, 2004). Unlike correlation-based distance functions, the Euclidean distance takes the magnitude of the expression data into account. It therefore preserves more information about the data and may be preferable (de Hoon et al., 2010). By using Euclidean distance as a measure of similarity, hyperspherical-shaped clusters of equal size are usually detected ( $\mathrm{Su} \& \mathrm{Chou}, 2001)$. In an $n$ dimensional population, the sum of squares between two points is computed by the following formula:

$$
d_{i j}^{2}=\sum_{k=1}^{n}\left(X_{i j}-X_{j k}\right)^{2}
$$

$X_{i j}$ and $\mathrm{X}_{\mathrm{jk}}$ denote the values on $i$ and $j$ coordinates of the variable $k$. If the objects are to be clustered according to different measurement units, variables must firstly be transformed to standard values and later squared Euclidean distances must be computed (Yaylalı, et al., 2006).

\section{Results}

This section explains the outcomes of the performance of the sectoral hierarchical clustering of SMEs in Turkey with respect to general supports for the years 2010 and 2011, and sets out the results of Ward's method. Because the concerned sectors have considerably lengthy denominations, the numbering of them was preferred. As the Ward's method dendogram in Figure 1 indicates, the sectors of SMEs were clearly agglomerated in two main clusters for the year 2010. The first cluster comprises Sectors 3, 9, 2, 6, 13, and 11; the second cluster comprises Sectors $4,10,1,5,7,12$, and 8 .

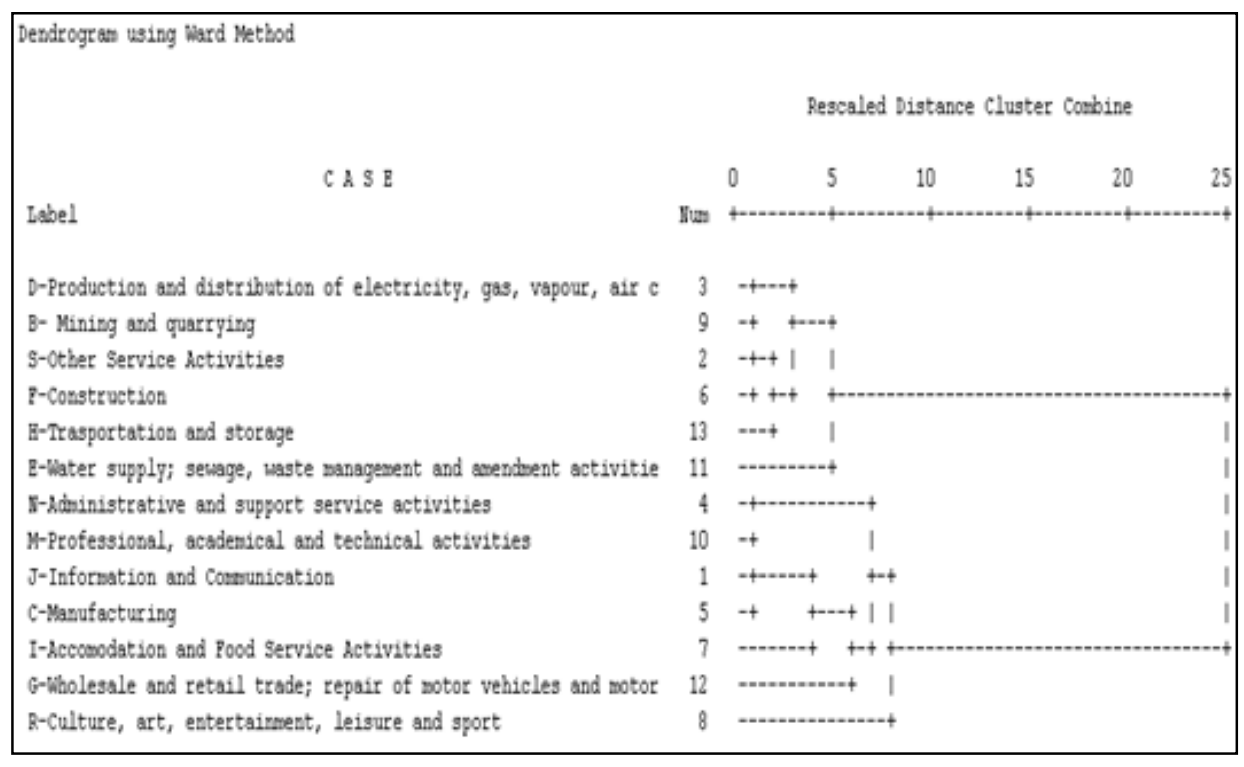

Figure 1. Ward's method dendogram (2010) 
The agglomerative schedule provides summary information about clusters with respect to the applied method as shown in Table 2. The left-hand side of the table sets out the research stage and all the clustered cases for the method concerned. Coefficients indicate the squared Euclidean distance between two cases; as the coefficients increase, the similarity between the two cases decreases. This means that two cases do not participate in a cluster. The right-hand side of the table exhibits at which stage the clusters resume until all of the cases agglomerate in one main cluster. The clustering process begins at Stage 1, where Sectors 3 and 9 are clustered. Later, these sectors cluster with Sector 2 at Stage 6. This stage indicated that the sectors would be clustered with Sector 11 at Stage 8. The process has ended at Stage 12, where all of the sectors for this cluster are agglomerated with Sector 1. This procedure was repeated for all stages, since all sectors were now clustered in a single cluster. Next, the researcher should confirm that the number of clusters observed on the Ward's method dendogram is ideal. This can be guaranteed by increasing the number of clusters to 3 and examining the results in that circumstance. Cluster membership is represented in Table 3. When we attempt to increase the number of clusters to 3, we see that Sector 8 is clustered in Cluster 3. This result was not optimum, because this sector tended to be involved with Cluster 1 in the centroid dendogram. As a result, we can suggest that the agglomeration of sectors in two clusters for Ward's method was ideal. The sectoral hierarchical clustering of SMEs in Turkey according to Ward's method for the year 2011 is represented in Figure 2. Ward's method agglomerated the sectors in three main clusters. The first cluster comprises Sectors 1, 4, 10, 8, 11, 3, and 9; the second cluster comprises Sectors 6, 13, and 7; the third cluster comprises Sectors 2, 12, and 5.

Table 2. Agglomeration schedule for ward's method (2010)

\begin{tabular}{|c|c|c|c|c|c|c|}
\hline \multirow[t]{2}{*}{ Stage } & \multicolumn{2}{|c|}{ Cluster Combined } & \multirow[t]{2}{*}{ Coefficients } & \multicolumn{2}{|c|}{ Stage Cluster First Appears } & \multirow[t]{2}{*}{ Next Stage } \\
\hline & Cluster 1 & Cluster 2 & & Cluster 1 & Cluster 2 & \\
\hline 1 & 3 & 9 & .186 & 0 & 0 & 6 \\
\hline 2 & 2 & 6 & .482 & 0 & 0 & 5 \\
\hline 3 & 1 & 5 & .946 & 0 & 0 & 7 \\
\hline 4 & 4 & 10 & 1.776 & 0 & 0 & 10 \\
\hline 5 & 2 & 13 & 2.921 & 2 & 0 & 6 \\
\hline 6 & 2 & 3 & 5.056 & 5 & 1 & 8 \\
\hline 7 & 1 & 7 & 7.517 & 3 & 0 & 9 \\
\hline 8 & 2 & 11 & 10.535 & 6 & 0 & 12 \\
\hline 9 & 1 & 12 & 14.129 & 7 & 0 & 10 \\
\hline 10 & 1 & 4 & 18.782 & 9 & 4 & 11 \\
\hline 11 & 1 & 8 & 23.925 & 10 & 0 & 12 \\
\hline 12 & 1 & 2 & 40.766 & 11 & 8 & 0 \\
\hline
\end{tabular}

Table 3. Clustering membership for ward's method (2010)

\begin{tabular}{lll}
\hline Case & 3 Clusters & 2 Clusters \\
\hline 1:J-Information and Communication & 1 & 1 \\
2:S-Other Service Activities & 2 & 2 \\
3:D-Production and distribution of electricity, gas, vapor, air conditioning & 2 & 2 \\
4:N-Administrative and support service activities & 1 & 1 \\
5:C-Manufacturing & 1 & 1 \\
6:F-Construction & 2 & 2 \\
7:I-Accomodation and Food Service Activities & 1 & 1 \\
8:R-Culture, art, entertainment, leisure and sport & 3 & 1 \\
9:B- Mining and quarrying & 2 & 2 \\
10:M-Professional, academic and technical activities & 1 & 1 \\
11:E-Water supply; sewage, waste management and amendment activities & 2 \\
12:G-Wholesale and retail trade; repair of motor vehicles and & 1 & 2 \\
motorcycles & & 1 \\
13:H-Trasportation and storage & 2 & 2 \\
\hline
\end{tabular}




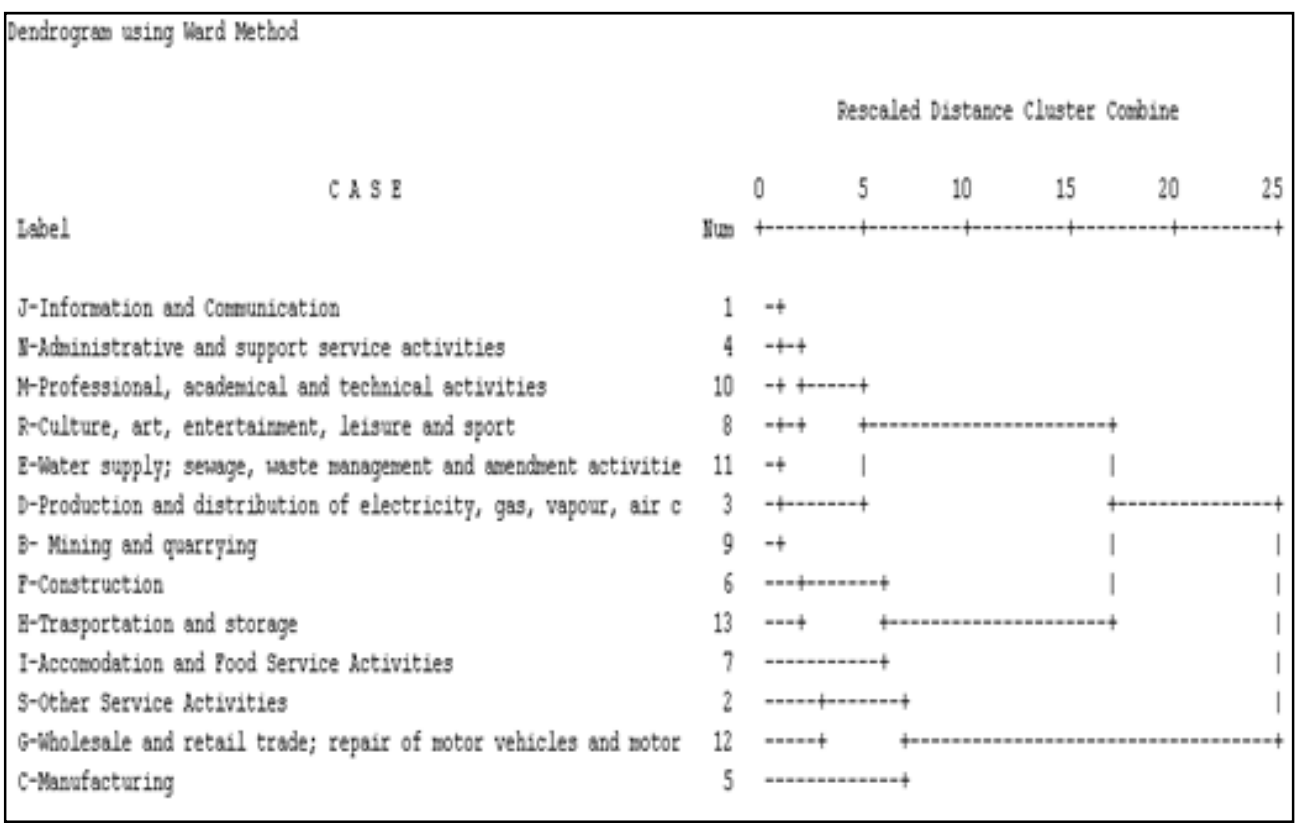

Figure 2. Ward's method dendogram (2011)

The examination of the agglomeration schedule for Ward's method as set out in Table 4 reveals that the clustering process begins at Stage 1, where Sector 1 and Sector 4 are clustered. These sectors are clustered with Sector 10 at Stage 4 and the process continues at Stage 6. Sector 8 is agglomerated with previously clustered sectors and with this we move to Stage 8. Sector 3 is involved in the cluster at Stage 8 and the process continues at Stage 11. Sector 6 is clustered at this stage, before moving to Stage 12. Finally, Sector 2 is agglomerated with all the other sectors in this process to form the first main cluster. The process is repeated until all the sectors are clustered in a single cluster. Subsequently we look for whether the number of clusters is ideal for the method. Cluster membership is investigated in Table 5 by increasing the number of clusters to 3 and decreasing to 2 . When the number of clusters is increased to 4, three sectors (Sector 6,7 and 13) are agglomerated in a distinct cluster. This solution was not optimum because these sectors tend to involve in the third cluster when the number of clusters is 3 . However, two clusters is not optimal, since one of the main clusters would be eliminated in this circumstance. As a result, it may be suggested that a three-cluster solution is ideal.

Table 4. Agglomeration schedule for ward's method (2011)

\begin{tabular}{rrrrrrrr}
\hline Stage & \multicolumn{2}{l}{ Cluster Combined } & Coefficients & \multicolumn{2}{c}{ Stage Cluster First Appears } & Next Stage \\
\cline { 2 - 3 } & Cluster 1 & \multicolumn{2}{c}{ Cluster 2 } & & Cluster 1 & Cluster 2 & \\
\hline 1 & 1 & 4 & .095 & 0 & 0 & 4 \\
2 & 3 & 9 & .214 & 0 & 0 & 8 \\
3 & 8 & 11 & .418 & & 0 & 0 \\
4 & 1 & 10 & .654 & & 1 & 0 & 6 \\
5 & 6 & 13 & 1.146 & & 0 & 0 & 9 \\
6 & 1 & 8 & 1.761 & 4 & 3 & 10 \\
7 & 2 & 12 & 2.511 & 0 & 0 & 11 \\
8 & 1 & 3 & 3.906 & 6 & 2 & 11 \\
9 & 6 & 7 & 5.669 & 5 & 0 & 12 \\
10 & 2 & 5 & 7.592 & 7 & 0 & 0 \\
11 & 1 & 6 & 12.610 & & 8 & 9 & 12 \\
12 & 1 & 2 & 20.263 & & 11 & 10 & 0 \\
\hline
\end{tabular}


Table 5. Clustering membership for ward's method (2011)

\begin{tabular}{lcccc}
\hline Case & & $\begin{array}{c}\mathbf{4} \\
\text { Clusters }\end{array}$ & $\begin{array}{c}\mathbf{3} \\
\text { Clusters }\end{array}$ & $\begin{array}{c}\mathbf{2} \\
\text { Clusters }\end{array}$ \\
\hline 1:J-Information and Communication & & 1 & 1 & 1 \\
2:S-Other Service Activities & 2 & 2 & 2 \\
3:D-Production and distribution of electricity, gas, vapor, air & 1 & 1 & 1 \\
conditioning & & 1 & 1 & 1 \\
4:N-Administrative and support service activities & & 3 & 2 & 2 \\
5:C-Manufacturing & 4 & 3 & 1 \\
6:F-Construction & 4 & 3 & 1 \\
7:I-Accomodation and Food Service Activities & 1 & 1 & 1 \\
8:R-Culture, art, entertainment, leisure and sport & 1 & 1 & 1 \\
9:B-Mining and quarrying & & 1 & 1 & 1 \\
10:M-Professional, academic and technical activities & & 1 & 1 & 1 \\
11:E-Water supply; sewage, waste management and amendment & & & \\
activities & & 2 & 2 & 2 \\
12:G-Wholesale and retail trade; repair of motor vehicles and & 2 & 3 & 1 \\
motorcycles & & 4 & 3 \\
13:H-Trasportation and storage & & &
\end{tabular}

Evolutionary economics may be considered as an attempt to look at an economic system, whether of the whole world or of its parts and in this sense, each economy may be seen as a segment of the larger evolutionary process of the universe (Boulding, 1991). Moreover, evoluationary theory involves a specification of the determinants of some equilavent of a notion of fitness implying the identification of a unit of selection and certain mechanisms through which the selection operates. Some analyses underlined the evolution of technology to suggest that some typical evolutionary patterns frequently at industry level (Dosi \& Nelson, 1994). Since evolutionary economics deals with the long-term processes of changing economic structures, more in particular with the increasing variety of technology and organizations, and the strategies to survive, regional policy makers usually suffer from dealing with the concerning changes (Lambooy \& Boschma, 2001). On the other hand, for the purposes of technology policy the principal themes of evolutionary economics are adopted as the processes which determine the range of actual innovations introduced into the economy and the processes which alter the relative economic importance of competing alternatives (Metcalfe, 1994). The results of this study can also be evaluated within the scope of this conceptual framework and industrial related variety argument. For both years being analyzed, there were related and unrelated clusters to survive. Technological and/or regional policies may affect the Turkish SME clusters with respect to evolutionary theory.

\section{Discussion and Conclusion}

In this study, a sectoral hierarchical clustering of SMEs in Turkey with respect to general support programs was performed for the years 2010 and 2011. Ward's hierarchical clustering method was used to interpret the data set obtained from KOSGEB headquarters. Ward's method offered a two-main-cluster solution for SMEs for the year 2010. With respect to their similarities, the sectors of first main cluster were: production and distribution of electricity, gas, vapor, air conditioning; mining and quarrying; other service activities; construction; transportation and storage; water supply; sewage, waste management, and amendment activities. The second main cluster included the following sectors: administrative and support service activities; information and communication; manufacturing; accommodation and food service activities; wholesale and retail trade; repair of motor vehicles and motor cycles; culture, art, entertainment, leisure and sport.

When the results of Ward's hierarchical clustering method for the year 2010 were examined, the suggestion arises that the rescaled distance clusters are different and so have introduced the similarities between sectors and their clustering expectations. When these distances were observed in detail and implementations established with respect to distances and similarities, more optimal decisions about the competitive advantage of SMEs could be made. In addition, because general support programs were initially introduced by KOSGEB in this year, the less competitive sectors in the Turkish economy could have experienced more challenges than the leading sectors and may not have realized the importance of these supports. So when these sectors have overcome their immediate 
challenges and been informed about the benefits of the programs, more competitive and more globalized SMEs will likely be created.

Ward's method offered a three-main-cluster solution for the year 2011. The sectors of first main cluster were information and communication; administrative and support service activities; professional, academic, and technical activities; culture, art, entertainment, leisure, and support; water supply, sewage, waste management, and amendment activities; production and distribution of electricity, gas, vapor, air conditioning; mining and quarrying. The second main cluster comprised the following sectors: construction; transportation and storage; accommodation and food service activities. The sectors of third main cluster were: other service activities; wholesale and retail trade; repair of motor vehicles and motorcycles.

When the results of Ward's hierarchical clustering method were considered for the year 2011, the suggestion arose that the number of main clusters has increased to three. This means that the dissimilarities between the clusters tend to decrease. The rescaled distance cluster should have been taken into account to determine the similarities or dissimilarities of the sectors and to interpret the results as a competitive advantage for SMEs. When compared to the previous year, the volume of general supports has significantly increased. In this manner, the less-supported sectors of the year 2010 were able to be involved in a cluster. Furthermore, it could be proposed that less competitive sectors may have overcome several challenges in the previous year and the gaps between leading sectors could have decreased.

The success of KOSGEB depends on the success of SMEs in Turkey. The global competitiveness of the enterprises plays a crucial role in KOSGEB's vision. The economic power of SMEs is the most important component that has to be taken into account for the organization to achieve its vision. The organization therefore has to monitor the economic conditions of SMEs on an ongoing basis and take action when needed. KOSGEB should consider the similarities with respect to rescaled distances and clusters of sectors to take advantage of general support programs and look for improved support opportunities to encourage these sectors by providing general support programs effectively and efficiently. The results of 2010 and 2011 can be used to lend assistance in future years of support. In addition, these results are able to serve as a model for other support programs because of central role that general supports play in all of KOSGEB's activities. Consequently, while evolutionary theory was consired in parallel with clustering results, technological and regional policies can be reshaped by the decision and policy makers.

\section{References}

Aldenderfer, M. S., \& Blashfield, R. K. (1984). Cluster analysis. Beverley Hills, CA: Sage Publications, Inc.

Altenburg, T., \& Meyer-Stamer, J. (1999). How to promote clusters: Policy experiences from Latin America, World Development, 27(9), 1693-1713. http://dx.doi.org/10.1016/S0305-750X(99)00081-9

Berry, A., Rodriguez, E., \& Sandee, H. (2001). Small and medium enterprised dynamics in Indonesia. Bulletin of Indonesian Economic Studies, 37(3), 363-384. http://dx.doi.org/10.1080/00074910152669181

Braun, P., McRae-Williams, P., \& Lowe, J. (2005). Small business clustering: Accessing knowledge through local network. Paper presented at the CRIC conference Beyond Cluster- Current Practices \& Future Strategies, June 30-July 1, in Ballarat, Australia.

Brenner, T. (2005). Local industrial clusters: Existence, emergence and Evolution. New York: Routledge Taylor $\&$ Francis Press.

Bresnahan, T., Gambardella, A., \& Saxenian, A. L. (2005). "Old economy" inputs for "new economy" outcomes: Cluster formation in the New Silicon Valleys. In S. Breschi, \& F. Malerba (Eds.), Clusters, Networks and Innovation (pp. 113-134). New York: Oxford University Press.

Bridges Jr., C. C. (1966). Hierarchical cluster analysis. Psychological Reports, 18, 851-854. http://dx.doi.org/10.2466/pr0.1966.18.3.851

Boulding, K. E. (1991). What is evolutionary economics? Journal of Evolutionary Economics, 1(1), 9-17. http://dx.doi.org/10.1007/BF01202334

Chen, X., \& Cao, L. (2006). SME clusters in China-One way to build up innovation capabilities. United Nations ESCAP. July-August 2006.

Çokluk, Ö., Şekercioğlu, G., \& Büyüköztürk, Ş. (2010). Multivariate statistics for social sciences: Applications of SPSS and Lisrel. Ankara: Pegem Academy.

de Hoon, M., Imoto, S., \& Miyano, S. (2010). The C clustering library. Tokyo: University of Tokyo. 
Dosi, G., \& Nelson, R. R. (1994). An introduction to evolutionary theories in economics. Journal of Evolutionary Economics, 4(3), 153-172. http://dx.doi.org/10.1007/BF01236366

Ferreira, M. P., Tavares, A. T., \& Hesterly, W. (2006). Evolution of industry clusters through spin-offs and the role of flagship firms. In A. T. Tavares, \& A. Teixeira (Eds.), Multinationals, Clusters and Innovation (pp. 87-106). New York: Palgrave Macmillan Press.

Härdle, W., \& Simar, L. (2007). Applied multivariate statistical analysis. New York: Springer Berlin Halderberg.

Izenmann, A. J. (2008). Modern multivariate statistical techniques: Regression, classification and manifold learning. Philadelphia: Springer Science+Business Media, LLC.

Johnson, R. A., \& Wichern, D. W. (2007). Applied multivariate statistical analysis. New Jersey: Pearson Education Inc.

Kalayc1, Ş. (2006). Multivariate statistical techniques using SPSS. Ankara: Baran Offset.

Kaplan, M. (2009). SMEs and clusters. MÜSİAD Çerçeve Dergisi, 17(51), 12-14. Retrieved from http://www.musiad.org.tr/img/yayinlarraporlar/cerceve_dergisi_51_03.pdf

Keeble, D., \& Nachum, L. (2002). Why do business service firms cluster? Small consultancies, clustering and decentralization in London and southern England. Transactions of the Institute of British Geographers, 27(1), 67-90. http://dx.doi.org/10.1111/1475-5661.00042

Ketels, C. H. M., \& Sölvell, Ö. (2006). Innovation clusters in the 10 new member states of the European Union. Europe Innova Paper No. 1, Luxembourg: Office for Official Publications of the European Communities.

Lambooy, J. G., \& Boschma, R. A. (2001). Evolutionary economics and regional policy. The Annals of Regional Science, 35(1), 113-131. http://dx.doi.org/10.1007/s001680000033

Landau, S., \& Everitt, B. S. (2004). A handbook of statistical analyses Using SPSS. London: Chapman \& Hall/CRC Press LLC.

Metcalfe, J. S. (1994). Evolutionary economics and technology policy. The Economic Journal, 104(425), 931-944. Retrieved from http://www.jstor.org/stable/2234988

Nordin, S. (2003). Tourism clustering \& innovation: Paths to economic growth \& development. European Tourism Research Institute Series 2003:14, Östersund: ETOUR.

Özdamar, K. (1999). Statistical data analysis using package programs: Multivariate Analyses-2. Eskişehir: Kaan Publishing.

Porter, M. E. (1990). The competitive advantage of nations. Free Press.

Porter, M. E. (2000). Systems of innovation: Growth, competitiveness and employment. Cheltenham: Edward Elgar Publishing Limited.

Rencher, A. C. (2002). Methods of multivariate analysis. New York: John Wiley \& Sons, Inc.

Republic of Turkey Ministry of Economy. (2011). SME Networking Project Executive Summary. Ankara.

Republic of Turkey Small and Medium Enterprises Development Organization. (2013). Vision/Mission. Retrieved September, 2013, from http://www.kosgeb.gov.tr/Pages/UI/Baskanligimiz.aspx?ref=71

Republic of Turkey Undersecretariat of Foreign Trade. (2008). White Book: Development of a clustering policy for Turkey. Ankara.

Rocha, H. O., \& Sternberg, R. (2005). Entrepreneurship: The role of clusters theoretical perspectives and empirical evidence from Germany. Small Business Economics, 24(3), 267-292. http://dx.doi.org/10.1007/s11187-005-1993-9

Shapira, P. (2008). Putting innovation in place: Policy strategies for industrial services, regional Clusters, and manufacturing SMEs in Japan and the United States. Prometheus: Critical Studies in Innovation, 26(1), 69-87. http://dx.doi.org/10.1080/08109020701846041

Sharma, S. (1996). Applied multivariate techniques. New York: John Wiley \& Sons, Inc.

$\mathrm{Su}$, M., \& Chou, C. (2001). A modified version of the K-Means algorithm with a distance based on cluster symmetry. IEEE Transactions on Pattern Analysis and Machine Intelligence, 23(6), 674-680. http://dx.doi.org/10.1109/34.927466

Tatlidil, H. (2002). Applied multivariate statistical analysis. Ankara: Ziraat Press. 
UNIDO. (2001). Development of clusters and networks of SMEs: The UNIDO Programme. Vienna: UNIDO.

Visser, E. (1999). A comparison of clustered and dispersed firms in the small-scale clothing industry of Lima. World Development, 27(9), 1553-1570. http://dx.doi.org/10.1016/S0305-750X(99)00077-7

Ward Jr., J. H. (1963). Hierarchical grouping to optimise an objective function. Journal of the American Statistical Association, 58, 236-244. Retrieved from http://iv.slis.indiana.edu/sw/data/ward.pdf

Wever, E., \& Stam, E. (1999). Clusters of high technology SMEs: The Dutch case. Regional Studies, 33(4), 391-400. http://dx.doi.org/10.1080/713693556

Wishart, D. (1969). An algorithm for hierarchical classification. Biometrics, 25(1), 165-170. Retrieved from http://www.jstor.org/stable/2528688

Xu, R., \& Wunsch II, D. C. (2009). Clustering. New Jersey: IEEE Press.

Yaylalı, M., Oktay, E., \& Akan, Y. (2006). Clustering of Turkish geographical regions and sectors of GDP with respect to per capita income. Atatürk University Journal of Graduate School of Social Sciences, 8(2), 311-334. Retrieved from http://e-dergi.atauni.edu.tr/index.php/SBED/article/view/368/362

\section{Copyrights}

Copyright for this article is retained by the author(s), with first publication rights granted to the journal.

This is an open-access article distributed under the terms and conditions of the Creative Commons Attribution license (http://creativecommons.org/licenses/by/3.0/). 